\title{
In vivo effects of nitrosyl hydrogen on cardiac function and sarcoplasmic reticulum calcium pump (SERCA2a) in rats with heart failure after myocardial infarction
}

\author{
Yanqing Guo ${ }^{1}$, Jiyao $\mathrm{Xu}^{1}$, Yongzhi Deng ${ }^{2}, \mathrm{Li} \mathrm{Wu}{ }^{1}$, Jingping Wang ${ }^{1}$, Jian $\mathrm{An}^{1}$ \\ ${ }^{1}$ Department of Cardiology, Shanxi Cardiovascular Hospital, Taiyuan, China; ${ }^{2}$ Department of Cardiovascular Surgery, Shanxi Cardiovascular \\ Hospital, Taiyuan, China \\ Contributions: (I) Conception and design: Y Guo; (II) Administrative support: J An; (III) Provision of study materials or patients: J Xu; (IV) Collection \\ and assembly of data: L Wu, J Wang; (V) Data analysis and interpretation: Y Deng; (VI) Manuscript writing: All authors; (VII) Final approval of \\ manuscript: All authors. \\ Correspondence to: Jian An. Department of Cardiology, Shanxi Cardiovascular Hospital, 18 Yifen street, Taiyuan 030024, China. \\ Email: anjianxxg@163.com.
}

\begin{abstract}
Background: Abnormal Ca2 + circulation in cardiomyocytes is an important cause of decreased myocardial contractility in failing hearts. Nitroxyl hydrogen (HNO) can oxidize $\mathrm{Ca}^{2+}$ cycle-related proteins, alter their biological functions, promote $\mathrm{Ca}^{2+}$ recovery as well as release, and enhance myocardial contractility. In this study, we aim to observe the effect of nitrosyl hydrogen (HNO) on the cardiac function of rats with heart failure and elucidate the underlying mechanism.
\end{abstract}

Methods: Twenty six male Wistar rats were randomly divided into heart failure group (HF group), Angeli's salt treatment group (HF + AS group) and sham operation group (Sham group). The HF + AS group rats were treated with HNO donor Angeli's salt by intraperitoneal injection of $1 \mathrm{mg} / \mathrm{kg} / \mathrm{d}$, and the rats in the HF group and the Sham group were given the same amount of normal saline. Cardiac function was measured by echocardiography before and after treatment. NT-proBNP was measured by enzyme immunoassay (ELISA) kit after treatment. Western blot were used to measure the expression of sarcoplasmic reticulum $\mathrm{Ca}^{2+}$-ATPase (SERCA) in protein levels in rats. The activities of SERCA2a were detected by the biochemical kit finally.

Results: We found that Nitrosyl hydrogen could significantly increase LVEF, $+\mathrm{dp} / \mathrm{dt},-\mathrm{dp} / \mathrm{dt}(\mathrm{P}<0.05)$, significantly decrease NT-ProBNP and LVEDP $(\mathrm{P}<0.01)$, and significantly enhance the activities of SERCA2a protein $(\mathrm{P}<0.05)$.

Conclusions: These findings suggest that Nitrosyl hydrogen could improve the cardiac function possibly by increasing protein activities of SERCA2a in rats.

Keywords: Nitroxyl; heart failure; hemodynamic; sarcoplasmic reticulum calcium pump

Submitted Feb 13, 2020. Accepted for publication Nov 08, 2020.

doi: $10.21037 / \mathrm{cdt}-20-201$

View this article at: http://dx.doi.org/10.21037/cdt-20-201

\section{Introduction}

Heart failure is the end stage of various diseases (1). It is a type of disease characterized by systolic or (and) diastolic dysfunction (2), and is one of the important reasons for the decline in quality of life and even death. Recent studies have found that abnormal $\mathrm{Ca}^{2+}$ circulation in cardiomyocytes is an important cause of decreased myocardial contractility in failing hearts (3-6). However, in myocardial depolarization, mitochondria release $\mathrm{Ca}^{2+}$ to the cytoplasm at a slower rate (3), which is also an important cause of the decrease in cytosolic $\mathrm{Ca}^{2+}$ concentration.

Recently, isolated hearts $(7,8)$ and cell experiments (9) 
have shown that Nitroxyl hydrogen (HNO) can oxidize $\mathrm{Ca}^{2+}$ cycle-related proteins, alter their biological functions, promote $\mathrm{Ca}^{2+}$ recovery as well as release, and enhance myocardial contractility. Our preliminary results have initially proved that $\mathrm{HNO}$ can enhance cardiac function and improve hemodynamics in HF rats, and may become a better HF treatment drug (10), but its specific mechanism of action remains unclear.

Through the intraperitoneal injection of HNO donor Angeli's salt (11) intervention in HF rats, this experiment followed the previous experimental method $(7,11)$, and detected peripheral blood $\mathrm{N}$-terminal B-type natriuretic peptide (NT-ProBNP) concentration, using cardiac ultrasound to detect blood flow dynamic changes (LVEF, LVFS, $\pm \mathrm{dp} / \mathrm{dt}, \mathrm{LVEDP}$ ), and the expression of cardiac tissue sarcoplasmic reticulum calcium pump as well as its biological activity. The results demonstrated that HNO could increase the activity of SERCA2a protein and protect the heart function of rats with heart failure after myocardial infarction. We present the study in accordance with the ARRIVE reporting checklist (available at http://dx.doi. org/10.21037/cdt-20-201).

\section{Methods}

\section{Experimental materials}

\section{Experimental animals}

Adult, healthy, and male Wistar rats, aged 6-8 weeks, weighing 160-180 g, purchased from the Experimental Animal Center of the Chinese Academy of Military Medical Sciences, Health No. 0011896.

The animals in this study were obtained from the Experimental Animal Center of the Chinese Academy of Military Medical Sciences. All animals handling and procedures were approved by the Ethics Committees of the Shanxi Cardiovascular Hospital, Taiyuan (Approval ID: 2017574), in compliance with institutional guidelines for the care and use of animals.

\section{Main equipment}

HX-100E ventilator (Chengdu Taimeng Company), BL410 Bioassay System (Chengdu Taimeng Company), Small Animal Ultrasound Instrument (GE Company), Analytical Balance (Shanghai Henggang Instrument Company), Spectrophotometer (Bio- Rad), PH (Bio-Rad), centrifuge (Thermo Forma), electrophoresis (Shanghai Tianneng Technology Co., Ltd.), gel imaging analyzer (Bio-Rad).

\section{Main experimental reagents}

Angeli's salt (Cayman), rat amino-terminal forebrain natriuretic peptide ELISA kit (Abnova), cell lysate, protease inhibitor, BCA protein concentration assay kit (all from Wuhan Boster), SERCA2a primary antibody (Abnova), goat anti-mouse secondary antibody (Abnova), $\beta$-actin primary antibody (Abnova), nitrocellulose membrane (Abnova), ATPase assay kit (Nanjing Jiancheng Institute of Bioengineering).

\section{Experimental methods}

Establishment of a model of myocardial infarction and heart failure in rats

Rats were weighed and anesthetized with $1 \%$ pentobarbital sodium $40 \mathrm{mg} / \mathrm{kg}$, prepared for skin, fixed on the back, intubated, and connected to an animal ventilator (tidal volume $10 \mathrm{~mL} / \mathrm{kg}$, respiratory rate 80 beats $/ \mathrm{min}$, breath ratio 1:1). Monitor the ECG. After conventional strong iodine disinfection, a transverse incision was made between the $3 \mathrm{rd}$ and 4th ribs on the left side of the sternum to expose the heart, and the left anterior descending coronary artery was ligated 2 to $3 \mathrm{~mm}$ below the left atrial appendage. Immediately after ligating, ECG monitoring showed that the R-wave amplitude of the limbs was significantly increased, and then the ST segments of I, II, III, avL, avF and $\mathrm{V} 3$ led up to $>0.2 \mathrm{mv}$, and showed a dynamic evolution process. At 1 week, the pathological $\mathrm{Q}$ wave was observed in the electrocardiogram for the myocardial infarction model. After 4 weeks of myocardial infarction, echocardiography showed that LVEF $\leq 45 \%$ was the successful sign of heart failure model (12-14).

\section{Experimental grouping and intervention}

In the experiment, 50 rats were selected, 42 of which were prepared for myocardial infarction heart failure model according to the above method. A total of 18 rats were successfully modeled 4 weeks after surgery, and were randomly divided into heart failure group (HF group, $\mathrm{n}=9$ ) and Angeli's salt treatment group (HF + AS group, $\mathrm{n}=9$ ). The remaining 8 were used as sham-operated groups (only a suture was placed under the left atrial appendage and no ligation was performed; Sham group, $n=8$ ). The $\mathrm{HNO}$ donor Angeli's salt of the rats in the HF + AS group (AS, vehicle was normal saline) were given $1 \mathrm{mg} / \mathrm{kg} / \mathrm{d}$ intraperitoneal injection (11). Both the HF group and the Sham group were given the same amount of physiological saline. At 2 weeks of intervention, 2 rats died in the HF 
group and 1 died in the HF+AS group. The cause of death was unknown. The rest of the rats had poor living conditions, markedly reduced activity, and shortness of breath. So, the experiment was over.

\section{Rat heart ultrasound examination}

The body weight of the rats was weighed by an electronic scale. Massage the abdomen until the rats were under deep anesthesia. The rats were fixed on the back and the skin was prepared in the chest area with curved scissors (up to the two forelimbs, down to the lower edge of the rib arch, left to the left front and rear limbs, right to the right sternal border). Disinfect the skin with iodophor. The small animal ultrasound probe (probe frequency $10 \mathrm{MHz}$, image depth $2.5 \mathrm{~cm}$ ) was used to measure the left ventricular long axis and short axis section of the heart, and the measurement parameters including left ventricular end diastolic diameter (LVEDd), left ventricular end systolic diameter (LVEDs), left ventricular ejection fraction (LVEF), and left ventricular short axis contraction rate (LVFS). Each index was measured 3 times, and the average value was used instead to observe the thickness of the ventricular wall and the movement.

\section{Detection of amino terminal brain natriuretic peptide precursor (NT-ProBNP)}

Two weeks after the intervention, blood was collected from the tail vein, serum was separated, and serum and standards were added to the plate containing the NT-ProBNP antibody. Incubate at $37^{\circ} \mathrm{C}$ for 1 hour, wash the plate 5 times, add the working solution for $30 \mathrm{~min}$ at $37^{\circ} \mathrm{C}$, wash the plate 5 times, add the developer, measure the standard and sample A values at $450 \mathrm{~nm}$, and calculate the NTProBNP content in the sample.

\section{Hemodynamic testing}

Open the BL-410 bio-detection system, select the hemodynamic detection interface, connect the pressure tube, flush the tube with heparin sodium and drain the air, and lubricate the catheter with paraffin oil. Massage the abdomen until the rat was under deep anesthesia. Free the right internal carotid artery, ligature the distal end of the artery, clamp the proximal end of the artery, use the ophthalmic scissors to make a inverted " $\mathrm{V}$ " incision in the middle of the artery, quickly insert the pressure catheter into the artery, and open the artery clip to observe the change of pressure waveform. Slowly introduce the catheter into the heart until a high pressure waveform appeared. After the waveform was stable, the left ventricular systolic pressure (LVSP), left ventricular end-diastolic pressure (LVEDP), the maximal rate of the increase of left room pressure $(+\mathrm{dp} / \mathrm{dt})$, and the maximal rate of the decrease of left room pressure $(-\mathrm{dp} / \mathrm{dt})$ were recorded. Each indicator was replaced by an average of 3 different moments.

\section{Heart tissue collection}

Open the rat thoracic cavity, venous blood was collected from the inferior vena cava and the heart was quickly removed, and washed with physiological saline for 3-5 times to clean the residual blood in the heart chamber. The left ventricular tissue of the left heart was freed, and the left ventricular non-infarcted myocardial tissue (excluding the myocardial infarction area and the infarct peripheral area) was collected and stored in liquid nitrogen for testing.

\section{Myocardial sarcoplasmic reticulum calcium pump protein expression}

(I) Tissue lysis: Extract total protein from left ventricular tissue, determinate protein concentration by Bradford method. (II) Sodium dodecyl sulfate-polyacrylamide gelation: $5 \%$ laminated glue $+10 \%$ separation gel. (III) Sodium dodecyl sulfate-polyacrylamide gel electrophoresis: a protein sample was aspirated by a micropipette and added to the well in a predetermined order, with glyceraldehyde3 -phosphate dehydrogenase (GAPDH) as an internal reference. (IV) Transfer film: nitrocellulose membrane, graphite electrode semi-dry rotation, transfer with constant current $1 \mathrm{~mA} / \mathrm{cm}^{2}$ gel electrophoresis for $1 \mathrm{~h}$. (V) Immunoblotting color development: The diluted primary antibody, SERCA (antibody titer 1:1,000), was added according to the antibody instructions. After $2 \mathrm{~h}$ at room temperature, the membrane was washed 3 times with Trimethylolamine Tween-20 buffer (TBST), and horseradish peroxidase-conjugated secondary antibody was added at 1:2,000, which was incubated at $37^{\circ} \mathrm{C}$ for $45 \mathrm{~min}$. ECLwas used, the film was scanned and imaged using an ODYSSEY 13.0 scanner. The absorbance analysis of the image was performed using the software Quantity one, and the ratio of the signal intensity of the target band to the internal reference band signal represented the expression level of the target receptor in the tissue.

\section{Sarcoplasmic reticulum calcium pump activity assay} The activity of sarcoplasmic reticulum calcium pump (SERCA2a) was determined by colorimetry using the SERCA2a enzyme kit from Nanjing Institute of 
Table 1 Comparison of parameters of echocardiography and NT-ProBNP before drug intervention in rats



Results are expressed as the mean $\pm \mathrm{SD}$. ${ }^{*} \mathrm{P}<0.01$ vs. sham. HF, heart failure; AS, Angeli's salt treatment; Sham, sham operation group; LVEDd, left ventricular end diastolic diameter; LVEDs, left ventricular end systolic diameter; LVEF, left ventricular ejection fraction; LVFS, left ventricular short axis contraction rate; NT-ProBNP, amino terminal brain natriuretic peptide precursor.

Bioengineering. Calcium pump activity assays were performed as described in the kit. SERCA2a activity = (sample OD value - control OD value) $\div$ standard OD value $\times$ standard concentration $\times$ sample dilution factor $\times 6$ $\div$ sample protein concentration.

\section{Statistical analysis}

All experimental data were analyzed using data processing software SPSS 24.0. Results were presented as means \pm SD for continuous variables in normal distribution. One-way analysis of variance (ANOVA) and Bonferroni correction were performed to compare the SHAM and HF+AS group to the HF group. $\mathrm{P}<0.05$ was considered statistically significant.

\section{Results}

At the 4th week after the establishment of heart failure model, drug intervention was started, and the experiment was finished 14 days after drug intervention.

\section{Comparison of basic values of rats in each group before drug intervention}

Before drug intervention, compared with Sham group, LVEDd, LVEDs and NT-ProBNP were significantly increased in both HF and HF+AS group $(\mathrm{P}<0.01)$; LVEF and LVFS were significantly decreased $(\mathrm{P}<0.01)$; compared with $\mathrm{HF}$ group, there were no significant differences in $\mathrm{HF}+\mathrm{AS}$ group regarding LVEDd, LVEDs, LVEF, LVFS, and NT-ProBNP in the rats $(\mathrm{P}>0.05)$ (Table 1, Figure 1$)$.

\section{Comparison of parameters of echocardiography and NT- ProBNP in rats after drug intervention}

After 2 weeks of drug intervention, compared with the HF group, LVEF of the HF+AS group was significantly increased $(\mathrm{P}<0.05)$, and the NT-ProBNP was significantly decreased $(\mathrm{P}<0.05)$. There was no significant difference between the LVFS, LVEDd and LVEDs $(\mathrm{P}>0.05)$. The results are shown in Figure 1 and Table 2.

\section{Comparison of hemodynamics in each group of rats}

After 2 weeks of drug intervention, compared with Sham group, LVEDP was significantly increased in HF group $(\mathrm{P}<0.01)$ and $\pm \mathrm{dp} / \mathrm{dt}$ were significantly decreased $(\mathrm{P}<0.01)$, and there was no significant difference in LVSP $(\mathrm{P}>0.05)$. Compared with the HF group, the LVEDP of the HF+AS group was significantly decreased $(\mathrm{P}<0.01)$ and $\pm \mathrm{dp} / \mathrm{dt}$ were significantly increased $(\mathrm{P}<0.05)$. There was no significant difference in LVSP between the two groups $(\mathrm{P}>0.05)$ (Table 3, Figure 2).

\section{Comparison of SERCA2 a protein expression and activities between groups}

After 2 weeks of drug intervention, the expression of SERCA2a protein in the HF group was decreased compared with the Sham group $(\mathrm{P}<0.01)$. Compared with the HF group, there was no significant difference in the expression of SERCA2a protein in the HF+AS group $(\mathrm{P}>0.05)$. After 2 weeks of drug intervention, the activity of SERCA2a protein in the HF group was decreased compared with the Sham group $(\mathrm{P}<0.01)$. Compared with the HF group, 

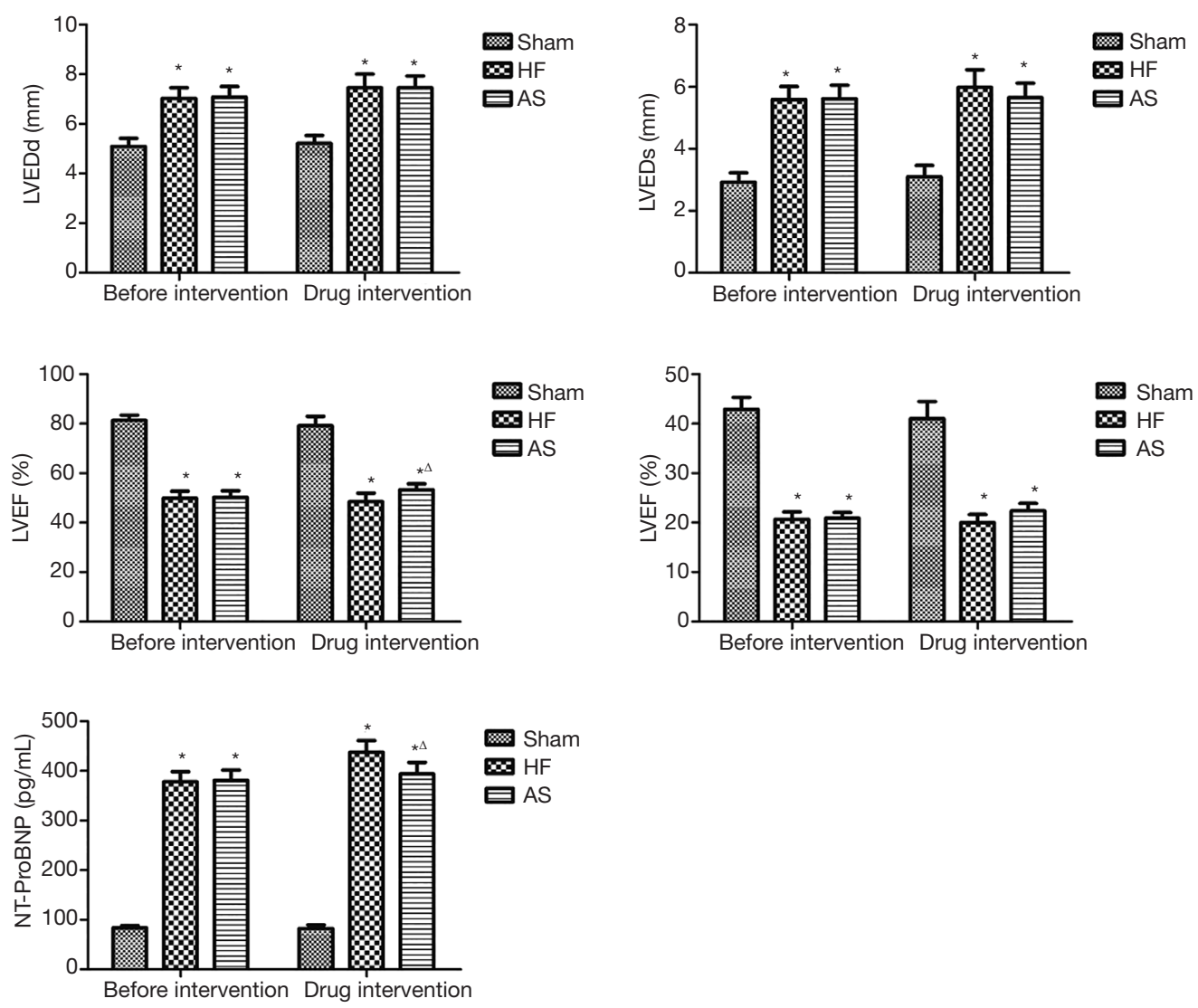

Figure 1 Effects of nitroxyl hydrogen on the changes of parameters of echocardiography and NT-ProBNP. Values are means \pm SD, ${ }^{*} \mathrm{P}<0.01$ vs. sham. ${ }^{\wedge} \mathrm{P}<0.05$ vs. HF.

Table 2 Comparison of parameters of echocardiography and NT-ProBNP after drug intervention in rats

\begin{tabular}{|c|c|c|c|}
\hline Parameters & Sham & $\mathrm{HF}$ & AS \\
\hline LVEDs (mm) & $3.10 \pm 0.36$ & $5.98 \pm 0.57^{*}$ & $5.65 \pm 0.47^{\star}$ \\
\hline LVEF (\%) & $79.13 \pm 3.80$ & $48.43 \pm 3.51^{*}$ & $53.25 \pm 2.43^{\star, \star \star}$ \\
\hline LVFS (\%) & $41.00 \pm 3.46$ & $20.00 \pm 1.63^{*}$ & $22.38 \pm 1.51^{*}$ \\
\hline HR (bpm) & $373.00 \pm 8.60$ & $388.29 \pm 11.28$ & $379.71 \pm 14.01$ \\
\hline
\end{tabular}

Results are expressed as the mean $\pm \mathrm{SD}$. ${ }^{*} \mathrm{P}<0.01$ vs. sham; ${ }^{*} \mathrm{P}<0.05$ vs. HF. HF, heart failure; AS, Angeli's salt treatment; Sham, sham operation group; LVEDd, left ventricular end diastolic diameter; LVEDs, left ventricular end systolic diameter; LVEF, left ventricular ejection fraction; LVFS, left ventricular short axis contraction rate; NT-ProBNP, amino terminal brain natriuretic peptide precursor. 
Table 3 Comparison of hemodynamics after drug intervention in rats

\begin{tabular}{lcccc}
\hline & LVSP $(\mathrm{mmHg})$ & LVEDP $(\mathrm{mmHg})$ & $+\mathrm{dp} / \mathrm{dt}(\mathrm{mmHg} / \mathrm{s})$ & $-\mathrm{dp} / \mathrm{dt}(\mathrm{mmHg} / \mathrm{s})$ \\
\hline Sham & $138.75 \pm 9.77$ & $7.18 \pm 0.91$ & $3950.13 \pm 264.29$ & $3842.25 \pm 241.07$ \\
HF & $132.86 \pm 8.93$ & $18.39 \pm 1.43^{*}$ & $2044.14 \pm 236.82^{*}$ & $1966.43 \pm 225.43^{*}$ \\
AS & $136.38 \pm 9.52$ & $15.48 \pm 1.23^{*, * \star}$ & $2412.00 \pm 357.35^{*, * \star}$ & $2302.25 \pm 335.54^{\star, * \star}$ \\
\hline
\end{tabular}

Results are expressed as the mean $\pm \mathrm{SD}$. ${ }^{\star} \mathrm{P}<0.01$ vs. sham; ${ }^{\star *} \mathrm{P}<0.05$ vs. HF. HF, heart failure; AS, Angeli's salt treatment; Sham, sham operation group; LVEDP, left ventricular end-diastolic pressure; LVSP, the left ventricular systolic pressure; $+\mathrm{dp} / \mathrm{dt}$, the maximal rate of the increase of left room pressure; - dp/dt, the maximal rate of the decrease of left room pressure.
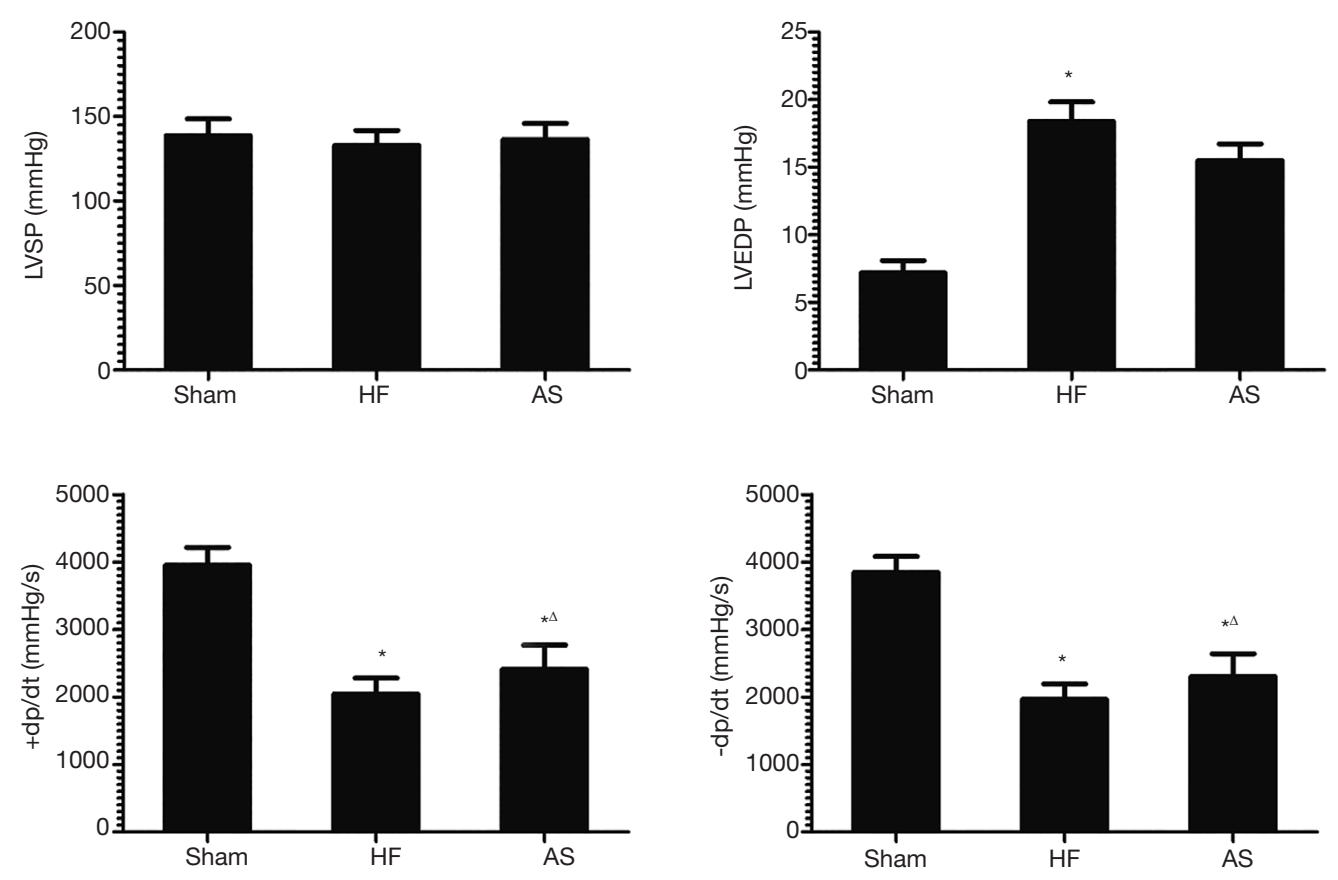

Figure 2 Effects of nitroxyl hydrogen on LVSP, LVEDP, $+\mathrm{dp} / \mathrm{dt},-\mathrm{dp} / \mathrm{dt}$. Values are means $\pm \mathrm{SD},{ }^{*} \mathrm{P}<0.01$ vs. sham. ${ }^{\Delta} \mathrm{P}<0.05$ vs. HF.

the activity of SERCA2a protein in the HF+AS group was significantly enhanced $(\mathrm{P}<0.05)$ (Figure 3).

\section{Discussion}

Nitroxyl hydrogen (HNO) is considered to be a single electron reduction product of nitric oxide (NO). It has more unique biochemical properties than other nitrogen oxides (15). In biological systems it can act as a reducing agent in combination with the metal reaction centers of some important proteins in the body (16) (such as reacting with $\mathrm{Fe}^{3+}$ in oxygenated hemoglobin and reducing it to $\mathrm{Fe}^{2+}$, or in superoxide dismutase $\mathrm{Cu}^{2+}$ is reduced to $\mathrm{Cu}^{+}$, etc.), and then their biological effects are changed to exert anti-oxidation and inhibit myocardial remodeling. HNO inhibits the production of reactive oxygen species. NADPH oxidase is one of the main sources of ROS production in cardiomyocytes and one of the important factors that trigger cardiomyocyte hypertrophy. In the failing heart, the uptake of $\mathrm{Ca}^{2+}$ by mitochondria is increased, and the calcium in mitochondria is overloaded, which leads to the disturbance of oxidative phosphorylation of cells and the increase of ROS production. The expression of NADPH oxidase subunit Nox2 is up-regulated during $\mathrm{HF}$, which is also an important reason for the increase of reactive oxygen species. In addition, HNO can downregulate the protein content of NADPH oxidase subunit Nox 2 in cardiomyocytes, thus reducing oxidative 

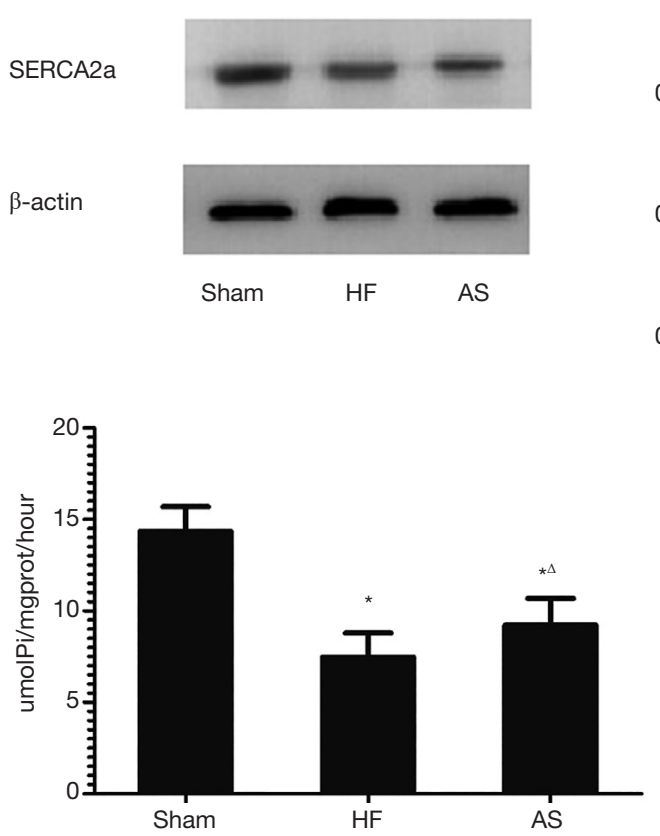

Figure 3 Effects of nitroxyl hydrogen on protein expression of SERCA2a. Values are means $\pm \mathrm{SD},{ }^{*} \mathrm{P}<0.01$ vs. sham. ${ }^{\Delta} \mathrm{P}<0.05$ vs. $\mathrm{HF}$.

stress (17). In addition, it can also be used as an oxidizing agent to react with sulfhydryl groups in the body to oxidize it to N-hydroxylated sulfinamide (RSNHOH) (18). If other sulfhydryl groups are present near the sulfhydryl group, disulfide bonds (19) can be formed, altering the protein conformation, resulting in changes in protein function, thereby improving cardiac function. Studies have shown that cysteine-reactive thiol groups in organisms play an important role in the redox reaction of HNO (20) and are also important groups involved in oxidative modification under physiological conditions. Cardiomyocytes involved in $\mathrm{Ca}^{2+}$ cycle-associated proteins contain cysteine-active sulfhydryl groups (20-23), so HNO may enhance systolic and diastolic function by modifying these specific thiol groups (24).

$\mathrm{HNO}$ is a transient substance that is chemically unstable. It is prone to dimerization and formation of secondary nitric acid $\left(\mathrm{H}_{2} \mathrm{~N}_{2} \mathrm{O}_{2}\right)$, which is subsequently decomposed into $\mathrm{N}_{2} \mathrm{O}$ and $\mathrm{H}_{2} \mathrm{O}$ (25). Therefore, pharmacological studies often use its donors for testing $(10,21)$. This experiment followed the previous method using the classical HNO donor Angeli's salt (AS, $\mathrm{Na}_{2} \mathrm{~N}_{2} \mathrm{O}_{3}$ ) for experiments $(8,11)$. It can be broken down into $\mathrm{HNO}$ and nitrite under physiological $\mathrm{pH}$ conditions. Because the vasodilation effect induced by HNO is 1,500 times that of nitrite (26), and its vasodilation

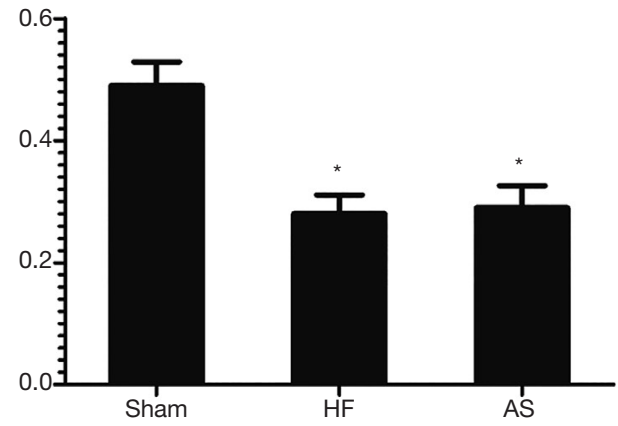


NT-ProBNP was significantly lower than that of heart failure group, indicating that the ventricular wall tension of $\mathrm{HF}$ rats was reduced. This again demonstrates from a biochemical point that HNO has an improved cardiac function in HF rats.

At the end of the experiment, we used a left heart pressure catheter to detect hemodynamic changes in each group. The experiment found that after administration of HNO donor AS, the LVEDP and $\pm \mathrm{dp} / \mathrm{dt}$ of $\mathrm{HF}$ rats were significantly improved, which was consistent with the experimental results of Chin et al. (7). Among them, $+\mathrm{dp} / \mathrm{dt}$ represents the maximum pressure rise rate of the left ventricle, reflecting the change of cardiac contractile function. In the experiment, we found that the AS treatment group $+\mathrm{dp} / \mathrm{dt}$ significantly increased compared with the heart failure group. indicating that $\mathrm{HNO}$ can increase the pressure development rate of the left ventricle, enhance myocardial contractility, and play a role in improving systolic function (26). $-\mathrm{dp} / \mathrm{dt}$ is the maximum pressure declining rate of the left ventricle, which mainly reflects the diastolic function of the heart. In the experimental group, the drug-treated group - dp/dt was elevated compared with the heart failure group. Therefore, HNO can also improve the diastolic function of $\mathrm{HF}$ rats (7). In addition, LVEDP, to some extent, can reflect the early heart function, heart load, etc. in HF. The results of this experiment show that HNO can reduce LVEDP in HF rats and protect heart function.

Cardiac sarcoplasmic reticulum calcium pump (SERCA2a) is an important structure for maintaining $\mathrm{Ca}^{2+}$ circulation in cardiomyocytes (4). In the repolarization of the myocardium, SERCA2a is activated, and most of the $\mathrm{Ca}^{2+}$ inverse concentration gradient in the cytoplasm is recovered into the sarcoplasmic reticulum for storage, resulting in a decrease in the concentration of $\mathrm{Ca}^{2+}$ in the cytoplasm, thereby relaxing the myocardial cells. Extracellular $\mathrm{Ca}^{2+}$ influx induces intracellular $\mathrm{Ca}^{2+}$ release from the sarcoplasmatic reticulum through the ryanodine receptor (Positive Feedback Loop) causing myocardial contraction. A sufficient amount of $\mathrm{Ca}^{2+}$ storage in the sarcoplasmic reticulum is an important factor in the enhancement of myocardial contractility (4).

In failing hearts, SERCA2a protein expression is reduced, biological activity is reduced, and is positively correlated with the severity of HF, which is considered to be an important cause of cardiac dysfunction. Studies have shown (30) that SERCA2a activity and protein expression are significantly reduced in the coronary heart disease $\mathrm{HF}$ rat model. In this experiment, the expression and activity of SERCA2a protein in HF rats were lower than those in the control group, which is consistent with this view. After 2 weeks of treatment with HNO donor AS, the activity of SERCA2a in the experimental rats was significantly increased. This may be because HNO forms a disulfide bond by oxidizing the cysteine active thiol group on SERCA2a (18), which changes the original spatial structure, enhances its biological function, and increases the $\mathrm{Ca}^{2+}$ recovery rate and the total sarcoplasmic reticulum $\mathrm{Ca}^{2+}$. It promotes $\mathrm{Ca}^{2+}$ circulation in cardiomyocytes (31), thereby improving cardiac function $(7,9,10,26)$. In addition, the expression level of SERCA2a protein is also an important factor affecting the $\mathrm{Ca}^{2+}$ cycle of cardiomyocytes, which directly affects the contractile function of the myocardium. However, the expression of SERCA2a protein in the cardiomyocytes of the drug-treated group did not increase significantly in this experiment, which could result from the fact that $\mathrm{HNO}$ exerts cardioprotective effects primarily by increasing the activity of SERCA2a protein.

Due to limited experimental conditions, the number of samples in this experiment was relatively small, and the duration of drug intervention was short. We did not set up multiple dose controls and monitor the heart rate, blood pressure fluctuations and changes in liver and kidney functions of rats. RT-PCR was not used to detect the expression of SERCA2a gene.

\section{Conclusions}

Most of the current research has been done in cell experiments or in vitro tissues, without elaborating on the specific roles they play in living organisms. In this experiment, HNO donor Angeli's salt was used to directly interfere with $\mathrm{HF}$ rat model to detect peripheral blood NT-ProBNP, cardiac ultrasound, hemodynamic changes and SERCA2a protein expression. It was preliminarily proved that HNO could increase cardiac ejection fraction, decrease peripheral blood NT-ProBNP level, and improve hemodynamics and enhance SERCA2a activity. This study provides theoretical evidence for the research of HF drugs and provides a new choice for the treatment of patients with HF. With the in-depth study of the mechanism of nitryl hydrogen drugs, its clinical application will be more extensive.

\section{Acknowledgments}

Funding: This study was funded by the basic applied 
research projects in Shanxi Province (grant number 201601D021156) and the Scientific Research Fund of Shanxi cardiovascular Hospital (grant number 20170203) and the Scientific Research Topics of Shanxi Health commission (grant number 2015070).

\section{Footnote}

Reporting Checklist: The authors have completed the ARRIVE reporting checklist. Available at http://dx.doi. org/10.21037/cdt-20-201

Data Sharing Statement: Available at http://dx.doi. org/10.21037/cdt-20-201

Peer Review File: Available at http://dx.doi.org/10.21037/ cdt-20-201

Conflicts of Interest: All authors have completed the ICMJE uniform disclosure form (available at http://dx.doi. org/10.21037/cdt-20-201). The authors have no conflicts of interest to declare.

Ethical Statement: The authors are accountable for all aspects of the work in ensuring that questions related to the accuracy or integrity of any part of the work are appropriately investigated and resolved. The animals in this study were obtained from the Experimental Animal Center of the Chinese Academy of Military Medical Sciences. All animals handling and procedures were approved by the Ethics Committees of the Shanxi Cardiovascular Hospital, Taiyuan (Approval ID: 2017574), in compliance with institutional guidelines for the care and use of animals.

Open Access Statement: This is an Open Access article distributed in accordance with the Creative Commons Attribution-NonCommercial-NoDerivs 4.0 International License (CC BY-NC-ND 4.0), which permits the noncommercial replication and distribution of the article with the strict proviso that no changes or edits are made and the original work is properly cited (including links to both the formal publication through the relevant DOI and the license). See: https://creativecommons.org/licenses/by-nc-nd/4.0/.

\section{References}

1. Gong HB, Wang L, Lv Q, et al. Improved systolic function of rat cardiocytes during heart failure by overexpression of
SERCA2a. Eur Rev Med Pharmacol Sci 2016;20:1590-6.

2. Davlouros PA, Gkizas V, Vogiatzi C, et al. Calcium Homeostasis and Kinetics in Heart Failure. Med Chem 2016;12:151-61.

3. Santulli G, Xie W, Reiken SR, et al. Mitochondrial calcium overload is a key determinant in heart failure. Proc Natl Acad Sci USA 2015;112:11389-94.

4. Sadredini M, Danielsen TK, Aronsen JM, et al. BetaAdrenoceptor Stimulation Reveals Ca2+ Waves and Sarcoplasmic Reticulum Ca2+ Depletion in Left Ventricular Cardiomyocytes from Post-Infarction Rats with and without Heart Failure. Plos One 2016;11:e0153887.

5. Izumi Y, Miura K, Iwao H. Therapeutic potential of vasopressin-receptor antagonists in heart failure. $\mathrm{J}$ Pharmacol Sci 2014;124:1-6.

6. Zhu XQ, Hong HS, Lin XH, et al. Changes in cardiac aldosterone and its synthase in rats with chronic heart failure: an intervention study of long-term treatment with recombinant human brain natriuretic peptide. Braz J Med Biol Res 2014;47:646-54.

7. Chin KY, Qin T, Cao N, et al. The concomitant coronary vasodilator and positive inotropic actions of the nitroxyl donor Angeli's salt in the intact rat heart: contribution of soluble guanylyl cyclase-dependent and -independent mechanisms. Br J Pharmacol 2014;171:1722-34.

8. Chin KY, Michel L, Qin CX, et al. The HNO donor Angeli's salt offers potential haemodynamic advantages over NO or dobutamine in ischaemia-reperfusion injury in the rat heart ex vivo. Pharmacol Res 2016;104:165-75.

9. Tocchetti CG, Wang W, Froehlich JP, et al. Nitroxyl improves cellular heart function by directly enhancing cardiac sarcoplasmic reticulum Ca2+ cycling. Circ Res 2007;100:96-104.

10. Kemp-Harper BK, Horowitz JD, Ritchie RH. Therapeutic Potential of Nitroxyl (HNO) Donors in the Management of Acute Decompensated Heart Failure. Drugs 2016;76:1337-48.

11. Choe CU, Lewerenz J, Fischer G, et al. Nitroxyl exacerbates ischemic cerebral injury and oxidative neurotoxicity. J Neurochem 2009;110:1766-73.

12. Liang T, Zhang Y, Yin S, et al. Cardio-protecteffect of qiliqiangxin capsule on left ventricular remodeling, dysfunction and apoptosis in heart failure rats after chronic myocardial infarction. Am J Transl Res 2016;8:2047-58.

13. Cheng S, Yu P, Yang L, et al. Astragaloside IV enhances cardioprotection of remote ischemic conditioning after acute myocardial infarction in rats. Am J Transl Res 
2016;8:4657-69.

14. Wei P, Yang XJ, Fu Q, et al. Intermedin attenuates myocardial infarction through activation of autophagy in a rat model of ischemic heart failure via both cAMP and MAPK/ERK1/2 pathways. Int J Clin Exp Pathol 2015;8:9836-44.

15. Fukuto JM, Jackson MI, Kaludercic N, et al. Examining Nitroxyl in Biological Systems. Methods Enzymol 2008;440:411-31.

16. Alexander SP, Benson HE, Faccenda E, et al. The Concise Guide to PHARMACOLOGY 2013/14: enzymes. Br J Pharmacol 2013;170:1797-867.

17. Guo Y, Xu J, Wu L, et al. Advances in research on treatment of heart failure with nitrosyl hydrogen. Heart Fail Rev 2019;24:941-8.

18. Keceli G, Moore CD, Labonte JW, et al. NMR detection and study of hydrolysis of HNO-derived sulfinamides. Biochemistry 2013;52:7387-96.

19. Kumar MR, Fukuto JM, Miranda KM, et al. Reactions of HNO with heme proteins: new routes to HNO-heme complexes and insight into physiological effects. Inorg Chem 2010;49:6283-92.

20. Fukuto JM, Carrington SJ. HNO signaling mechanisms. Antioxid Redox Signal 2011;14:1649-57.

21. Tocchetti CG, Stanley BA, Murray CI, et al. Playing with cardiac "redox switches": the "HNO way" to modulate cardiac function. Antioxid Redox Signal 2011;14:1687-98.

22. Meissner G. Regulation of Ryanodine Receptor Ion Channels Through Posttranslational Modifications. Curr Top Membr 2010;66:91-113.

Cite this article as: Guo Y, Xu J, Deng Y, Wu L, Wang J, An J. In vivo effects of nitrosyl hydrogen on cardiac function and sarcoplasmic reticulum calcium pump (SERCA2a) in rats with heart failure after myocardial infarction. Cardiovasc Diagn Ther 2020;10(6):1795-1804. doi: 10.21037/cdt-20-201
23. Ge Y, Moss RL. Nitroxyl, redox switches, cardiac myofilaments, and heart failure: a prequel to novel therapeutics. Circ Res 2012;111:954-6.

24. Sivakumaran V, Stanley BA, Tocchetti CG, et al. HNO enhances SERCA2a activity and cardiomyocyte function by promoting redox-dependent phospholamban oligomerization. Antioxid Redox Signal 2013;19:1185-97.

25. DuMond JF, King SB. The chemistry of nitroxyl-releasing compounds. Antioxid Redox Signal 2011;14:1637-48.

26. Favaloro JL, Kemp-Harper BK. The nitroxyl anion (HNO) is a potent dilator of rat coronary vasculature. Cardiovasc Res 2007;73:587-96.

27. Andrews KL, Sampson AK, Irvine JC, et al. Nitroxyl (HNO) reduces endothelial and monocyte activation and promotes M2 macrophage polarization. Clin Sci (Lond) 2016;130:1629-40.

28. Zhu G, Groneberg D, Sikka G, et al. Soluble guanylate cyclase is required for systemic vasodilation but not positive inotropy induced by nitroxyl in the mouse. Hypertension 2015;65:385-92.

29. De Witt BJ, Marrone JR, Kaye AD, et al. Comparison of responses to novel nitric oxide donors in the feline pulmonary vascular bed. Eur J Pharmacol 2001;430:311-5.

30. Wang YL, Fan XY, Liu GP, et al. Effect of sarcoplasmic reticulum calcium pump on excitation-contraction coupling function in cardiomyocytes with heart failure. $\mathrm{J}$ Electron Microsc 2010;29:486-90.

31. Arcaro A, Lembo G, Tocchetti CG. Nitroxyl (HNO) for treatment of acute heart failure. Curr Heart Fail Rep 2014;11:227-35. 